\title{
Long-term variable controlled ventilation in severe acute respiratory distress syndrome - an experimental randomized controlled study
}

Güldner A., Kiss T., Bluth T., Braune A., Koch T., Gama de Abreu M.

Department of Anaesthesiology and Intensive Care Medicine, University Hospital Carl Gustav Carus,

Technische Universität Dresden, Dresden, Germany

\section{Rationale}

- Mechanical ventilation with variable tidal volumes (VT) is able to improve lung function and attenuate ventilator induced lung injury (VILI) in acute respiratory distress syndrome (ARDS). Previous investigations on variable ventilation have been limited to mild or moderate ARDS and short periods of mechanical ventilation. [1].

- We hypothesized that $24 \mathrm{~h}$ of variable ventilation improves lung function and reduces VILI in experimental severe ARDS.

\section{Methods}

- 14 juvenile pigs were anesthetized, intubated, and mechanically ventilated.

- ARDS was induced using a double hit consisting of saline lung lavage followed by injurious ventilation.

- Random assignment to one of two groups: 1) non-variable volume controlled ventilation (VCV), 2) variable ventilation (Noisy) with random $V_{T}$-variation with a coefficient of variation of $V_{T}$ was $30 \%$. $V_{T}$ (VCV) and mean $V_{T}$ (Noisy) $6 \mathrm{ml} / \mathrm{kg}$

- PEEP was titrated according to the table by the ARDS network

- Animals were ventilated supine for 24 hours.

- Lung aeration was determined by CT, PET was used to assess lung regional [18F] fluorodeoxyglucose ([18F]FDG) uptake, as well as the distribution of perfusion by $68 \mathrm{Ga}$ labelled microspheres following induction of ARDS (day 1) and 24h thereafter (day 2).

- Gas exchange and respiratory system mechanics were determined every 4 hours

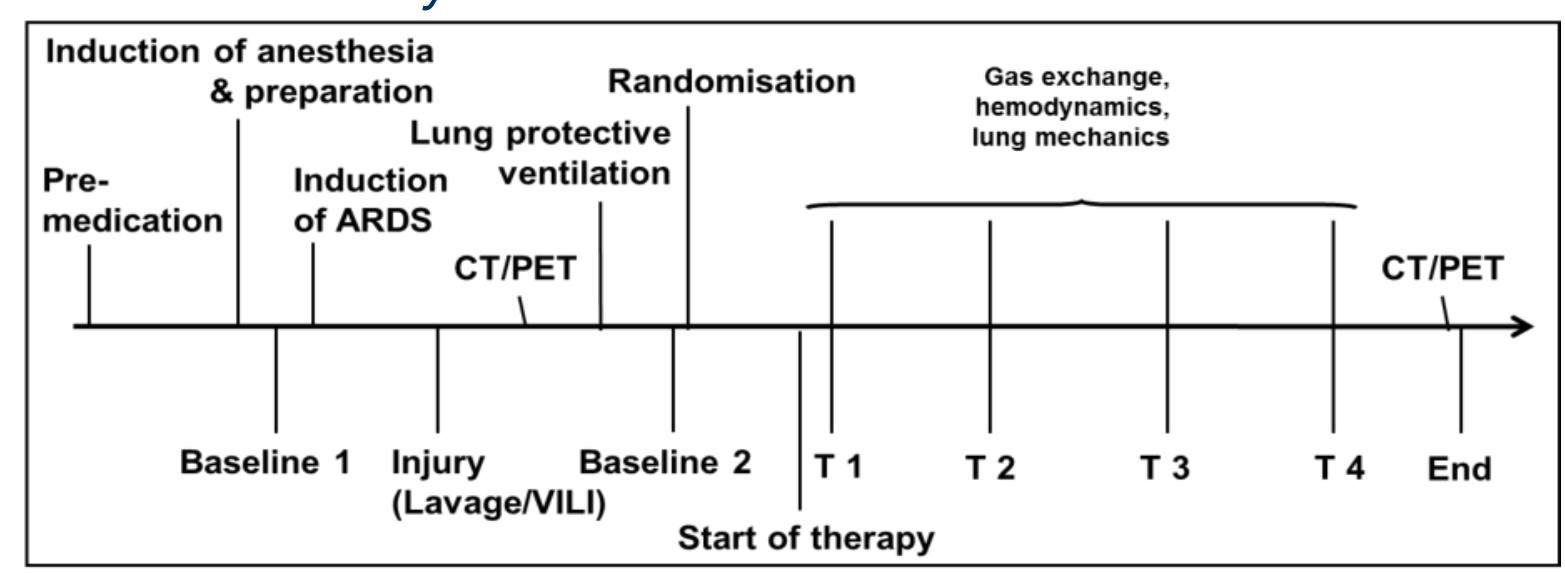

Fig. 1: Time course of interventions

\section{Results}

- The specific uptake rate $\mathrm{K}_{\text {is }}$ of [18F]FDG increased on day 2 in all lung regions in both groups.

- A lower amount of normally-aerated lung tissue was detected on day 2 in both groups.

- Perfusion was redistributed towards ventral and cranial lung regions on day 2 in both groups.

- Lung mechanics, gas exchange and hemodynamics did not differ between groups.

\section{Conclusion}

Compared to conventional, variable controlled ventilation with PEEP titrated according to the ARDS network neither enhanced lung function, nor reduced lung inflammation after $24 \mathrm{~h}$ of mechanical ventilation.

\section{References}

[1] Huhle et al: Variable ventilation from bench to beside. Crit. Care, 20(1), DOI: 10.1186/s13054-016-1216-6, 2016.

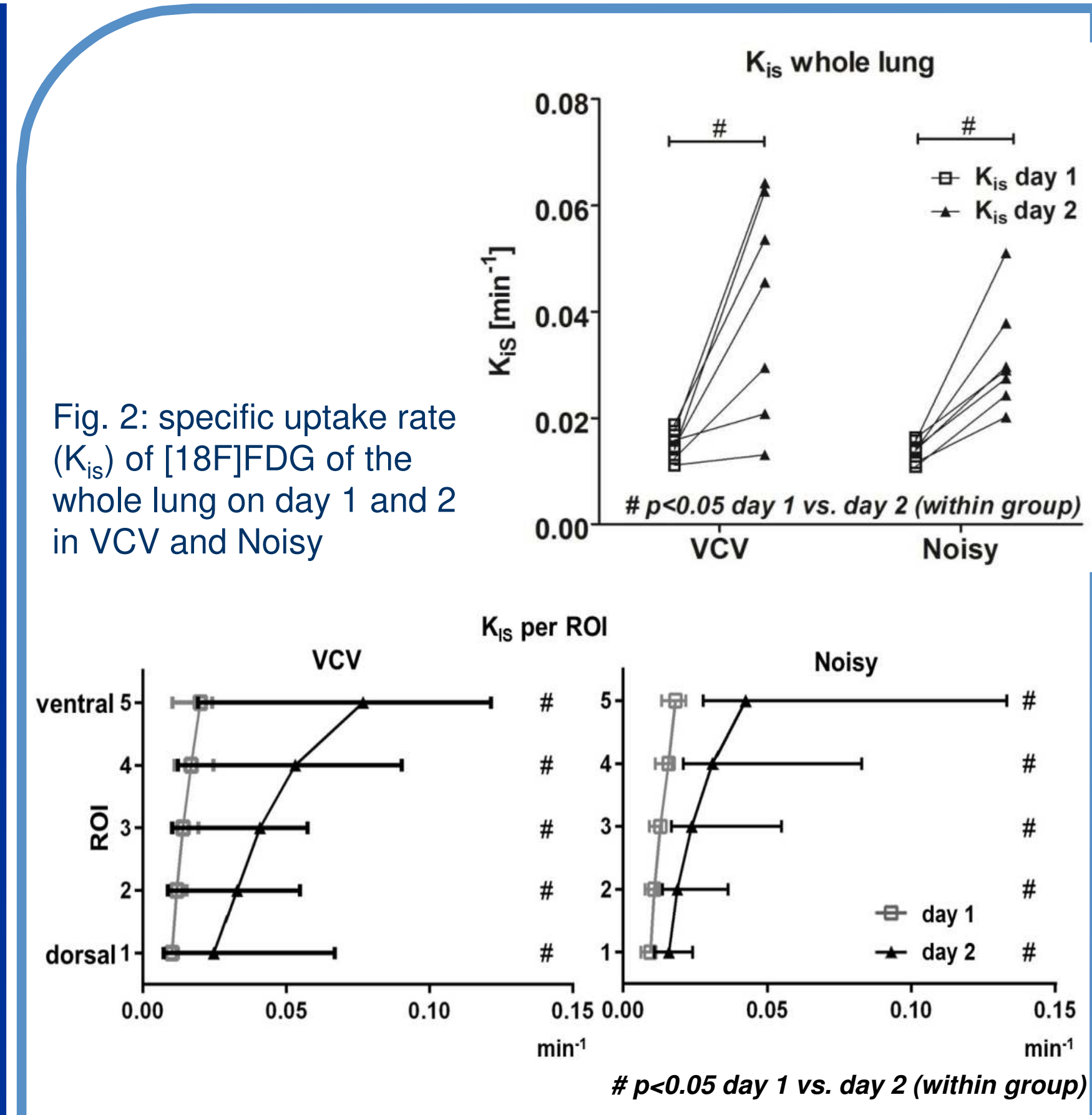

Fig. 3: specific uptake rate (Kis) of [18F]FDG in five regions of interest (ROI) from ventral to dorsal on day 1 and 2 in VCV and Noisy; median and range

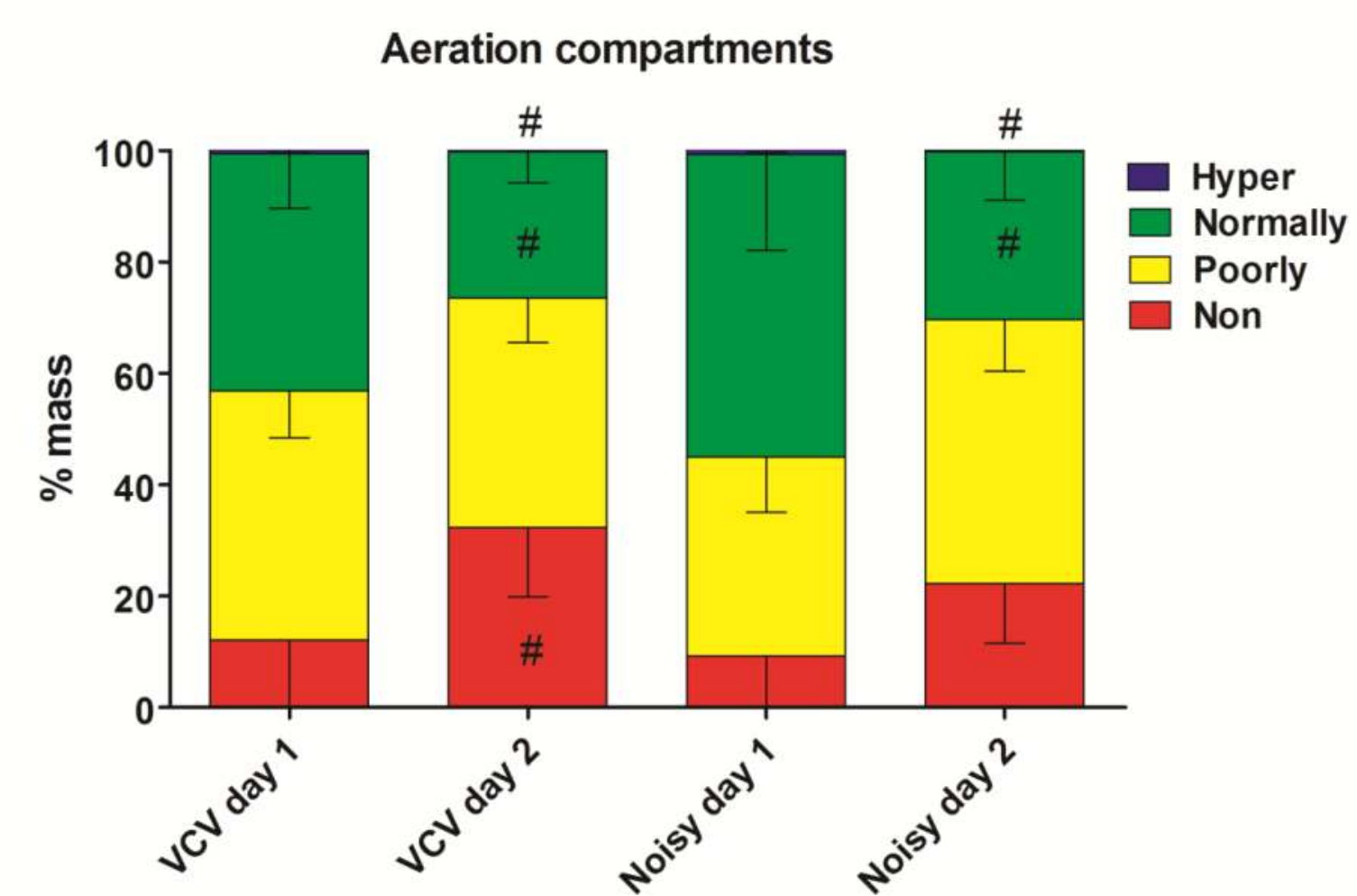

Fig. 4: Aeration compartments in \% of lung mass on day 1 and 2 in VCV and Noisy; median and range

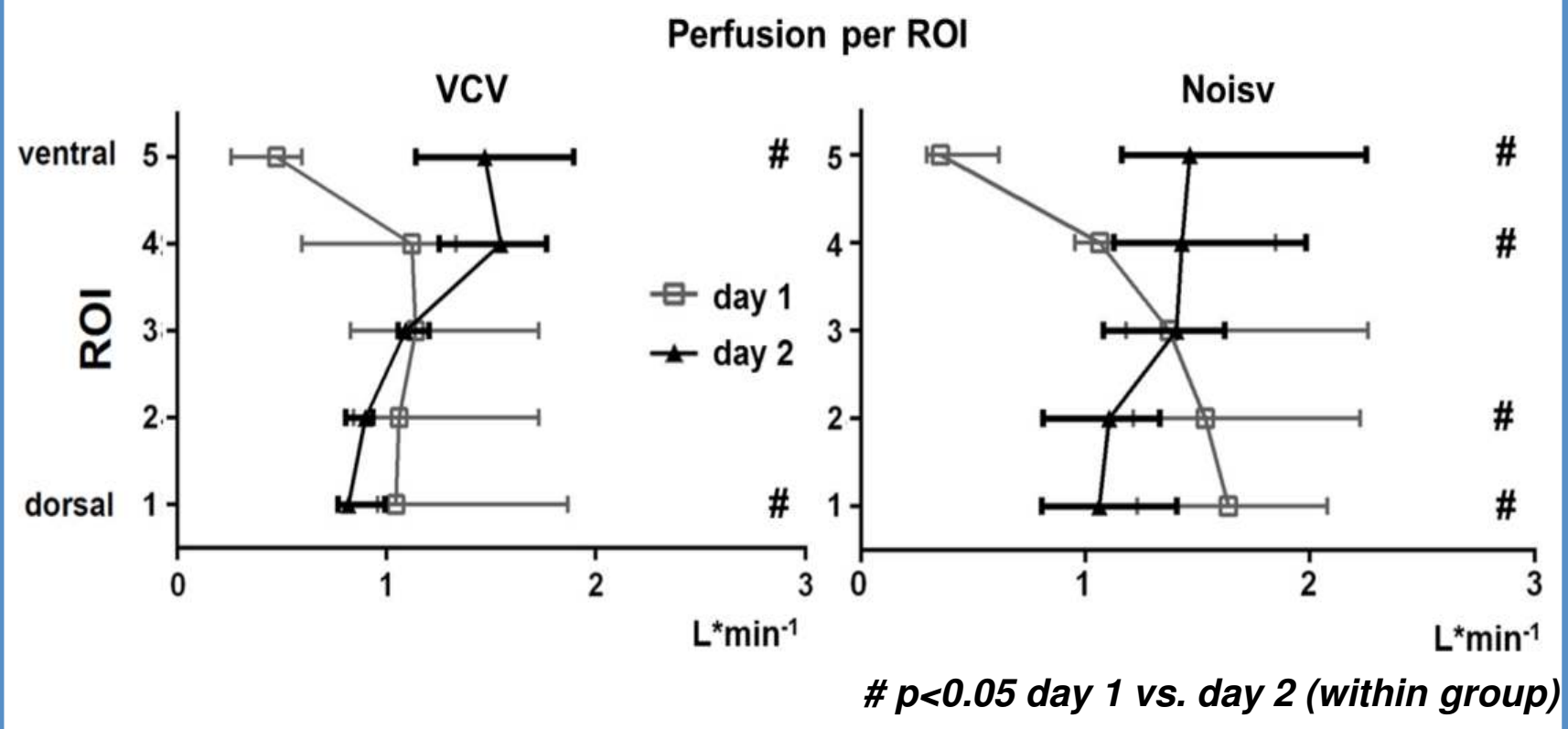

Fig. 5: Perfusion in five regions of interest $(\mathrm{ROI})$ from ventral to dorsal on day 1 and 2 in VCV and Noisy; median and range 